\title{
Generalizing the Ryu-Takayanagi formula to probe entanglement shadows of BTZ black holes
}

\author{
Marius Gerbershagen ${ }^{a}$ \\ ${ }^{a}$ Institut für Theoretische Physik und Astrophysik \\ and Würzburg-Dresden Cluster of Excellence ct.qmat, \\ Julius-Maximilians-Universität Würzburg, Am Hubland, 97074 Würzburg, Germany \\ E-mail: marius.gerbershagen@physik.uni-wuerzburg.de
}

We define a generalized entanglement measure in the context of $\mathrm{AdS}_{3} / \mathrm{CFT}_{2}$. Compared to the ordinary entanglement entropy between spatial degrees of freedom dual to the area of the RyuTakayanagi surface, we take into account both entanglement between spatial degrees of freedom as well as between different fields of the boundary CFT. We then calculate this generalized entanglement measure in a thermal state dual to the BTZ black hole in the setting of the D1/D5 system at and close to the orbifold point. We find that the entanglement entropy defined in this way is dual to the length of a geodesic with non-zero winding number. Such geodesics probe the entire bulk geometry, including regions known as entanglement shadows which are not reached by any Ryu-Takayanagi surface. This allows us to describe regions close to the black hole horizon in the one-sided black hole and the wormhole growth in the case of a two-sided black hole from entanglement data in the boundary field theory, leading us to propose that entanglement is in fact enough to reconstruct the full BTZ geometry from boundary data.

\footnotetext{
*** The European Physical Society Conference on High Energy Physics (EPS-HEP2021), *** ***26-30 July $2021 * * *$

*** Online conference, jointly organized by Universität Hamburg and the research center DESY ***
} 


\section{Introduction}

The study of entanglement in the AdS/CFT correspondence has provided remarkable insights into the nature of this duality as well as aspects of quantum gravity in general, starting from the seminal work of Ryu and Takayanagi relating the entanglement entropy of a subregion $A$ in the boundary CFT to the area of a minimal codimension two surface $\gamma_{A}$ in the bulk AdS space [1],

$$
S_{A}=-\operatorname{Tr}\left(\rho_{A} \log \rho_{A}\right)=\frac{\operatorname{Area}\left(\gamma_{A}\right)}{4 G_{N}} .
$$

An important open question regarding the role of entanglement in AdS/CFT is whether entanglement data of the boundary CFT is enough to describe the entire bulk spacetime dual to a particular CFT state. While the connection between entanglement entropy of a spatial subregion and a geometric quantity in the bulk clearly shows that entanglement and geometry are connected (this is often referred to as "entanglement builds geometry" or "it from qubit", see e.g. [2]), there are certain regions of the bulk spacetime termed entanglement shadows which are inaccessible to any RyuTakayanagi surface $\gamma_{A}$ [3-5]. These occur for instance around naked singularities or black hole horizons.

In the following, we introduce a generalized entanglement measure whose bulk dual probes entanglement shadows of BTZ black holes. This new entanglement measure takes into account entanglement between spatial degrees of freedom as well as between different fields of the theory.

Generalized entanglement measures in this spirit have been studied previously in $\mathrm{AdS}_{3} / \mathrm{CFT}_{2}$ for states dual to conical defects in the bulk under the name of "entwinement" [5-8]. There, it was found that entwinement is dual to the length of a geodesic - distinct from any RT surface - winding a non-zero number of times around the conical defect. These geodesics probe arbitrarily close to the conical singularity, allowing for a description of the entanglement shadow using entanglement data in the CFT.

\section{The $S_{N}$ orbifold CFT of the D1/D5 system}

Concretely, we study our generalized entanglement measure in the D1/D5 system at the orbifold point, which we now introduce. The D1/D5 system is a particular top down $\mathrm{AdS}_{3} / \mathrm{CFT}_{2}$ construction (see [9] for a review), where the field content of the theory is known in detail. This is a necessary condition in order to define entanglement between different fields, which is why we use this specific model instead of a generic bottom-up instance of AdS/CFT.

Furthermore, we consider the system in the limit where the CFT is weakly coupled and dual to a strongly coupled bulk theory. At this so-called orbifold point, the boundary CFT is given by a $S_{N}$ orbifold theory constructed in the following way: take $N$ copies of a fixed "seed CFT"1 and identify the copies under the $S_{N}$ permutation symmetry, that is only allow states which are $S_{N}$ invariant. In the following, we also take the large central charge limit where $N \rightarrow \infty$ and we only work to leading order in $N$.

In addition to the Hilbert space of states of the $N$ copies of the seed theory symmetrized under permutations, consistency requirements such as modular invariance of the thermal partition

\footnotetext{
${ }^{1}$ For the D1/D5 system, this is a supersymmetric theory of four free bosons and fermions with target space $T^{4}$. However all of our calculations are independent of the choice of seed theory, therefore we leave it unspecified.
} 
function also enforce the introduction of twisted sectors. These sectors contain states in which the fields of the theory (collectively denoted by $X^{i}$ for $i \in\{1, \ldots, N\}$ ) obey boundary conditions which are periodic up to permutations,

$$
X^{i}(\phi+2 \pi)=X^{g(i)}(\phi) \quad \text { for } g \in S_{N} .
$$

The twisted sectors are labeled by conjugacy classes $C$ of the $S_{N}$ group which are specified by the number $n_{m}$ of permutation cycles of length $m$ in the group elements of the given conjugacy class. The total length of the cycles is given by the number of copies of the seed theory, $N=\sum_{m} m n_{m}$.

\section{Generalized entanglement entropy}

Let us now introduce the generalized entanglement measure which we are working with. As a reminder, the ordinary entanglement entropy as used in the RT formula is defined as follows. First, choose a spatial subregion $A$ and split the Hilbert space into tensor factors belonging to the degrees of freedom in $A$ and its complement, $\mathcal{H}=\mathcal{H}_{A} \otimes \mathcal{H}_{A^{c}}$. Then, calculate the reduced density matrix $\rho_{A}=\operatorname{Tr}_{\mathcal{H}_{A c}}(\rho)$ whose von Neumann entropy defines the entanglement entropy $S_{A}=-\operatorname{Tr}\left(\rho_{A} \log \rho_{A}\right)$. Our generalized entanglement measure differs from this definition in the following two points:

1. We consider a subset $\left\{C_{i}\right\}$ of the twisted sectors of the $S_{N}$ orbifold. Due to the orthogonality of states in different twisted sectors, the thermal density matrix is block diagonal, $\rho(\beta)=$ $\bigoplus_{C} p_{C} \rho_{C}$, where $\rho_{C}$ is the contribution of the twisted sector $C$ (normalized such that $\operatorname{Tr} \rho_{C}=1$ ). We consider a part of the thermal density matrix made up of multiple such blocks $C_{1}, \ldots, C_{r}$, that is we restrict to the density matrix $\rho_{\left\{C_{i}\right\}}$ defined by

$$
\rho_{\left\{C_{i}\right\}}=\bigoplus_{i=1}^{r} p_{C_{i}} \rho_{C_{i}}
$$

2. We consider entanglement between different fields as well as between spatial degrees of freedom. For the ordinary entanglement entropy, the degrees of freedom consist of all fields localized in the same subregion $A$. Here, we consider different fields $X^{i}$ localized in different subregions $A^{i}$. For these degrees of freedom, we can again define a reduced density matrix by tracing out the complement ${ }^{2}$,

$$
\rho_{\left\{A_{i}\right\},\left\{C_{i}\right\}}=\operatorname{Tr}_{\left\{A_{i}\right\}^{c}}\left(\rho_{\left\{C_{i}\right\}}\right) .
$$

The generalized entanglement entropy is then defined as the von Neumann entropy of $\rho_{\left\{A_{i}\right\},\left\{C_{i}\right\}}$,

$$
S_{\left\{A_{i}\right\},\left\{C_{i}\right\}}=-\operatorname{Tr}\left(\rho_{\left\{A_{i}\right\},\left\{C_{i}\right\}} \log \rho_{\left\{A_{i}\right\},\left\{C_{i}\right\}}\right) .
$$

Of course, this definition gives a very large number of possible entanglement measures depending on the choice of ingredients $\left\{C_{i}\right\}$ and $\left\{A_{i}\right\}$. To find one whose bulk dual is a simple geometric

\footnotetext{
${ }^{2}$ Taking this trace is somewhat subtle in the $S_{N}$ orbifold since the physical space of gauge invariant (i.e. $S_{N}$ invariant) states does not factorize into a tensor product (see [8] and references therein for details).
} 
quantity, we use the following choices. Fix a number $n \in \mathbb{N}$ and choose $\left\{C_{i}\right\}$ such that for each $i, C_{i}$ only contains cycles whose length $m$ is a multiple of $n^{3}$. Moreover, we take $n$ to be of order $O\left(N^{0}\right)$ in the large $N$ limit. For the subset $\left\{A_{i}\right\}$, we consider $w$ fields $X^{i_{1}}(\phi \in[0,2 \pi]), \ldots, X^{i_{w}}(\phi \in[0,2 \pi])$ in the full space together with a single field $X^{i_{w+1}}(\phi \in[0, L])$ in some spatial subregion $[0, L]$, chosen such that the all of the fields $X^{i_{1}}, \ldots, X^{i_{w+1}}$ are continuously connected by the twisted boundary conditions (that is, $\left.X^{i_{k}}(\phi+2 \pi)=X^{i_{k+1}}(\phi)\right)$. Each of the $k$ groups of $n$ fields inside a cycle of length $m=k n$ of the twisted boundary conditions contains one such subset of $w+1$ fields.

To calculate the entanglement entropy for this choice of ingredients, we use standard replica trick techniques: we first calculate the Rényi entropy

$$
S_{\left\{A_{i}\right\},\left\{C_{i}\right\}}^{(\alpha)}=\frac{1}{1-\alpha} \log \operatorname{Tr} \rho_{\left\{A_{i}\right\},\left\{C_{i}\right\}}^{\alpha}
$$

for integer $\alpha$ and then analytically continue to $\alpha \rightarrow 1$ to obtain (5). The Rényi entropy is determined from the partition function on a (higher genus) replica surface constructed by gluing together $\alpha$ copies of the system along the entangling intervals determined by $\left\{A_{i}\right\}^{4}$. This partition function decomposes into conformal blocks and due to features of the spectrum and OPE coefficients of the $S_{N}$ orbifold CFT coming from its holographic nature is dominated by a single conformal block [10].

The conformal blocks depend on two conformal dimensions coming from the inserting a projection operator onto a Virasoro primary and its descendants into the replica partition function. We can think of these projection operators as non-local operators encircling a topologically nontrivial cycle of the replica manifold. One such cycle lies around either the temporal or spatial circle of one of the $\alpha$ tori of the replica manifold ${ }^{5}$, while the other lies around either the entangling interval branch cut or its complement. Different choices of where to put this projection operator correspond to different channels in the conformal block decomposition. For holographic CFTs, the channel in which the conformal block with lowest scaling dimensions is the smallest dominates.

For the ordinary entanglement entropy, the dominant conformal block is the vacuum block where the conformal dimensions of all operators vanish. For the generalized entanglement entropy, the leading contribution in the dominant channel at low temperatures comes from the ground state of the $(n)^{N / n}$ twisted sector with conformal weight $h=\bar{h}=\frac{c}{n 24}(n-1 / n)$ due to the restriction to a subset $\left\{C_{i}\right\}$ of the twisted sectors projecting out the contribution of the untwisted sector containing the vacuum state. On the other hand, the conformal block in the dominant channel at high temperatures is unaffected by the restriction onto the subset of twisted sectors. This can be seen by noting that for high temperatures, where this channel is dominant, all twisted sectors contribute to the leading order (in $N$ ) result of the thermal partition function while at low temperature the leading contribution comes from the untwisted sector only. The scaling dimension of the other operator in the conformal block vanishes in the dominant channel.

${ }^{3}$ In the case that $n$ is not a divisor of $N$, the $C_{i}$ are allowed to contain $O\left(N^{0}\right)$ cycles of arbitrary length. These cycles don't influence the leading order in $N$ of the entanglement measure we are computing, therefore in the following we take w.l.o.g. $n$ to be a divisor of $N$.

${ }^{4}$ In principle, we have different entangling intervals for each field $X^{i}$. However, for the choice of $\left\{A_{i}\right\}$ that we are using, we can model the entangling interval as a single interval winding $w$ times around the spatial circle of each of the $\alpha$ tori. Due to the restriction to the subset of twisted sectors, all fields obey boundary conditions that effectively fuse $n$ fields together into a single field on a torus with a spatial circle of length $2 \pi n$, therefore this entangling interval is well-defined.

${ }^{5}$ Equivalently, we can think of this projection operator as restricting the sum over states in the thermal density matrix. 
Finally, the conformal blocks themselves are computed from a monodromy method following $[11,12]$, giving for small winding numbers $w$

$$
S_{\left\{A_{i}\right\},\left\{C_{i}\right\}}=\left\{\begin{array}{l}
\frac{c}{3 n} \log \left[\frac{n}{\epsilon} \sin \left(\frac{\pi(w+L)}{n}\right)\right], \beta>2 \pi n, \\
\frac{c}{3 n} \log \left[\frac{\beta}{2 \pi \epsilon} \sinh \left(\frac{2 \pi^{2}(w+L)}{\beta}\right)\right], \beta<2 \pi n .
\end{array}\right.
$$

For temperatures above the BTZ black hole threshold, $\beta<2 \pi$, the result is equal to the length of a geodesic with opening angle $2 \pi L$ winding $w$ times around the black hole horizon. For lower temperatures where the dual bulk geometry is given by thermal $\mathrm{AdS}_{3}$, the result is equal to a geodesic with opening angle $2 \pi(w+L) / n$ for $\beta>2 \pi n$, while for $2 \pi<\beta<2 \pi n$, no geometric interpretation of our result in therms of a geodesic in the dual bulk geometry exists. For large $w$ and large temperatures, there is a phase transition analogous to the ordinary entanglement entropy. In this case, the result for the generalized entanglement entropy is equal to the BTZ black hole entropy plus the length of a geodesic with opening angle $2 \pi(1-L)$ and winding number $n-w-1$.

Finally, we note that a straightforward generalization to the two-sided black hole gives a generalized entanglement entropy (measuring entanglement between different asymptotic boundaries) dual to the length of two geodesics stretching through the wormhole for early times and two geodesics with winding number $w$ on opposite boundaries of the wormhole for late times. The transition between these two regimes happens at a critical time that is proportional to the winding number.

\section{Discussion}

Let us note first that the bulk dual of the generalized entanglement measure we have defined is able to probe the entire (classical) bulk geometry of the BTZ black hole up to the horizon in the one-sided case and even including the behind the horizon region in the two-sided case. For $N \rightarrow \infty$, we can make the winding number as large as we want by suitably choosing the degrees of freedom for which we calculate the entanglement entropy. The dual geodesics then cover the entire entanglement shadow.

This assertion is of course somewhat premature since the calculation presented here was done at the orbifold point where the dual gravity theory is strongly coupled and a classical bulk geometry is not a good approximation of the gravity dynamics. However, we expect our generalized entanglement measure to be invariant under deformations away from the orbifold point, at least up to subleading corrections of order $O\left(N^{0}\right)$. One indication for this comes from the fact that for the ordinary entanglement entropy, which is a special case of the more general setup studied here, the RT formula in the supergravity limit gives the same result as what we obtain at the orbifold point. Moreover, we have checked that our results are invariant up to subleading corrections to second order in perturbation theory.

Another comment is on the interpretation of the restriction onto a subset of the twisted sectors in the gravity theory at the orbifold point, which is the well-studied tensionless limit of string theory in the D1/D5 system (see e.g. [13-15] and references therein). In particular, the gravity partition function on a BTZ background has been obtained in this system [16]. By explicit comparison with the $S_{N}$ orbifold partition function, it is possible to show that the restriction to particular twisted 
sectors corresponds to allowing only toroidal worldsheets with particular winding numbers around the spatial circle of the boundary torus.

\section{References}

[1] S. Ryu and T. Takayanagi, Holographic derivation of entanglement entropy from AdS/CFT, Phys. Rev. Lett. 96 (2006) 181602 [hep-th/0603001].

[2] M. Van Raamsdonk, Building up spacetime with quantum entanglement, Gen. Rel. Grav. 42 (2010) 2323 [1005 . 3035].

[3] V.E. Hubeny, H. Maxfield, M. Rangamani and E. Tonni, Holographic entanglement plateaux, JHEP 08 (2013) 092 [1306 . 4004].

[4] B. Freivogel, R. Jefferson, L. Kabir, B. Mosk and I.-S. Yang, Casting Shadows on Holographic Reconstruction, Phys. Rev. D91 (2015) 086013 [1412 . 5175].

[5] V. Balasubramanian, B.D. Chowdhury, B. Czech and J. de Boer, Entwinement and the emergence of spacetime, JHEP 01 (2015) 048 [1406. 5859].

[6] V. Balasubramanian, A. Bernamonti, B. Craps, T. De Jonckheere and F. Galli, Entwinement in discretely gauged theories, JHEP 12 (2016) 094 [1609.03991].

[7] V. Balasubramanian, B. Craps, T. De Jonckheere and G. Sárosi, Entanglement versus entwinement in symmetric product orbifolds, JHEP 01 (2019) 190 [1806.02871].

[8] J. Erdmenger and M. Gerbershagen, Entwinement as a possible alternative to complexity, JHEP 03 (2020) 082 [1910. 05352].

[9] J.R. David, G. Mandal and S.R. Wadia, Microscopic formulation of black holes in string theory, Phys. Rept. 369 (2002) 549 [hep-th/0203048].

[10] T. Hartman, C.A. Keller and B. Stoica, Universal Spectrum of $2 d$ Conformal Field Theory in the Large c Limit, JHEP 09 (2014) 118 [1405 . 5137].

[11] T. Hartman, Entanglement Entropy at Large Central Charge, 1303.6955.

[12] M. Gerbershagen, Monodromy methods for torus conformal blocks and entanglement entropy at large central charge, 2101.11642.

[13] M.R. Gaberdiel and R. Gopakumar, Tensionless string spectra on AdS $S_{3}$, JHEP 05 (2018) 085 [1803.04423].

[14] L. Eberhardt, M.R. Gaberdiel and R. Gopakumar, The Worldsheet Dual of the Symmetric Product CFT, JHEP 04 (2019) 103 [1812.01007].

[15] L. Eberhardt, M.R. Gaberdiel and R. Gopakumar, Deriving the $A d S_{3} / C F T_{2}$ correspondence, JHEP 02 (2020) 136 [1911.00378].

[16] L. Eberhardt, Partition functions of the tensionless string, JHEP 03 (2021) 176 [2008.07533]. 